University of Nebraska - Lincoln

DigitalCommons@University of Nebraska-Lincoln

Faculty Publications from Nebraska Center for Materials and Nanoscience, Nebraska Center Materials and Nanoscience

2020

SVAT4: a computer program for visualization and analysis of crystal structures

Xingzhong Li

Follow this and additional works at: https://digitalcommons.unl.edu/cmrafacpub

Part of the Atomic, Molecular and Optical Physics Commons, Condensed Matter Physics Commons, Engineering Physics Commons, Graphics and Human Computer Interfaces Commons, and the Other

Physics Commons

This Article is brought to you for free and open access by the Materials and Nanoscience, Nebraska Center for (NCMN) at DigitalCommons@University of Nebraska - Lincoln. It has been accepted for inclusion in Faculty Publications from Nebraska Center for Materials and Nanoscience by an authorized administrator of DigitalCommons@University of Nebraska - Lincoln. 


\section{SVAT4: a computer program for visualization and analysis of crystal structures}

\section{X.-Z. Li}

J. Appl. Cryst. (2020). 53, 848-853

\section{IUCr Journals CRYSTALLOGRAPHY JOURNALS ONLINE}

Copyright (C) International Union of Crystallography

Author(s) of this article may load this reprint on their own web site or institutional repository provided that this cover page is retained. Republication of this article or its storage in electronic databases other than as specified above is not permitted without prior permission in writing from the IUCr.

For further information see https://journals.iucr.org/services/authorrights.html 


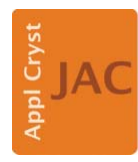

JOURNAL OF

APPLIED

CRYSTALLOGRAPHY

ISSN 1600-5767

Received 3 February 2020

Accepted 2 April 2020

Edited by S. Boutet, SLAC National Accelerator Laboratory, Menlo Park, USA

Keywords: crystal structure visualization; crystal structure analysis; computer programs.

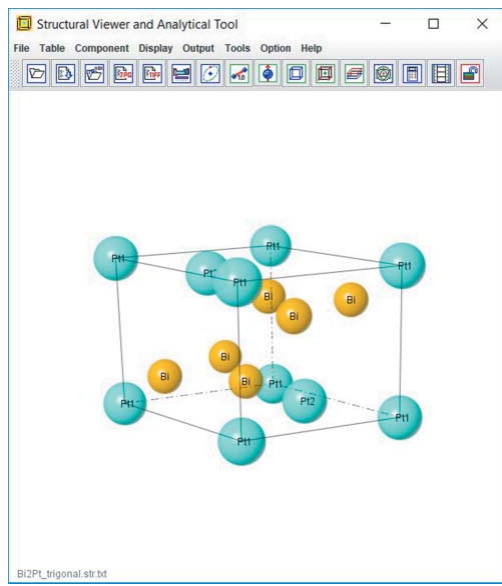

(C) 2020 International Union of Crystallography

\section{SVAT4: a computer program for visualization and analysis of crystal structures}

\author{
X.-Z. Li*
}

Nebraska Center for Materials and Nanoscience, University of Nebraska, Lincoln, NE 68588, USA. *Correspondence e-mail: xzli@unl.edu

SVAT4 is a computer program for interactive visualization of three-dimensional crystal structures, including chemical bonds and magnetic moments. A wide range of functions, e.g. revealing atomic layers and polyhedral clusters, are available for further structural analysis. Atomic sizes, colors, appearance, view directions and view modes (orthographic or perspective views) are adjustable. Customized work for the visualization and analysis can be saved and then reloaded. SVAT4 provides a template to simplify the process of preparation of a new data file. SVAT4 can generate high-quality images for publication and animations for presentations. The usability of SVAT4 is broadened by a software suite for simulation and analysis of electron diffraction patterns.

\section{Introduction}

Visualization of crystal structures and interpretation of functional atomic clusters is essential in research and education on metallography, crystallography and other related research fields. Thus, various software is available for these purposes (see http://www.ccp14.ac.uk/solution/structuredrawing/index. html and https://www.rcsb.org/pages/thirdparty/molecular graphics), either as commercial products or free for the scientific community, some of it in open-source forms.

SVAT was initially developed for visualization of crystal structures in 2011: the need for yet more software for such purposes was due to the growth of the software components and the number of users of the Landyne software suite for electron diffraction simulation and crystallographic analysis $(\mathrm{Li}, 2016,2020 a, b)$. More functions, as requested by users, were gradually integrated into SVAT for visualization and analysis of crystal structures. The current version is SVAT4, which exists as a component in the Landyne software suite and also works as standalone software.

SVAT and follow-up versions have been used for nearly ten years in the author's home institute and by users of the Landyne suite. So far, most applications of SVAT4 involved alloys or intermetallic compounds in materials science research. Although SVAT4 is lightweight, it provides some unique features beyond the standard functions as a structural visualization and analysis tool. The limitations of SVAT4 are, for example, that it is not suitable for handling large molecules, and it has no functions to show atoms as ellipsoids or to create a stereo pair.

This paper reports on the current status of SVAT4 and its relation to other components in the Landyne software suite. SVAT4 was developed in Java. The Java Runtime Environment, or JRE, must be installed in order to run SVAT4. Java Advanced Imaging is needed for generating TIFF images. The SVAT4 executable codes, a collection of input data and a user 


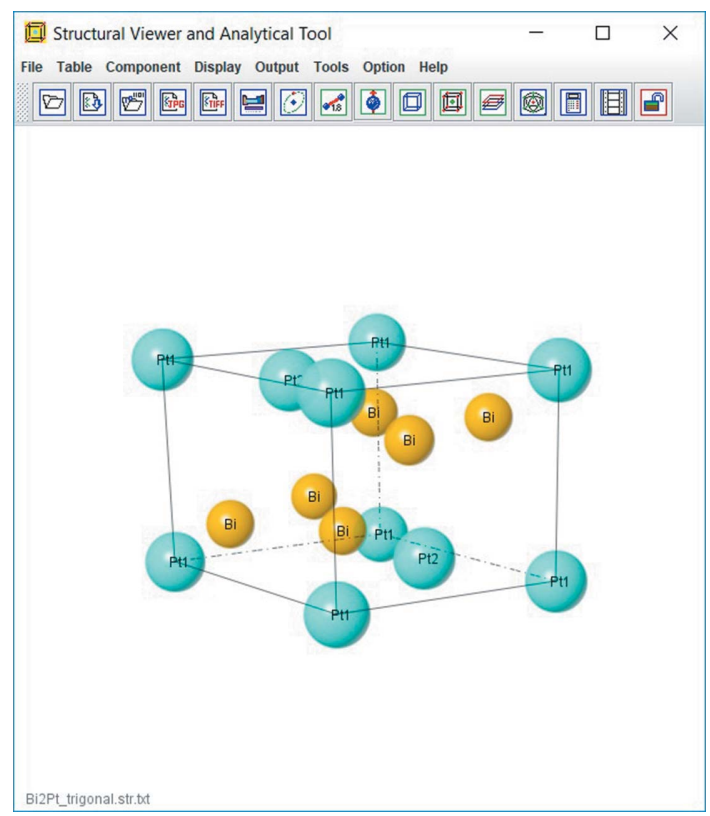

Figure 1

The GUI of $S V A T 4$, with the $\mathrm{Bi}_{2} \mathrm{Pt}$ structure as an example. manual can be downloaded from the author's web pages $(\mathrm{Li}$, $2020 a, b)$.

\section{Design and functions}

\subsection{Software design}

The graphical user interface (GUI) of SVAT4 was built from the Java Swing package. It consists of a drop-down menu, a menu bar, a display panel (as shown in Fig. 1) and multiple operational panels (as shown in Fig. 2). The drop-down menu provides access to all the operational panels, while the menu bar lists only frequently used ones. The display panel and a control panel are designed to be always on screen, while other operational panels can be switched on and off.

With the inclination toward lightweight software, 3D vision is generated using 3D geometrical calculation and Java2D functions instead of the Java3D library. In addition to the essential functions for an interactive 3D visualization of crystal structure in a unit cell, including chemical bonds and magnetic moments, a wide range of functions for analysis have been implemented, e.g. structural projection or layer-by-layer visualization along a user-defined $[u v w]$ direction and visualization as polyhedral clusters. Atoms in a central unit cell and adjacent unit cells are stored for the construction of the

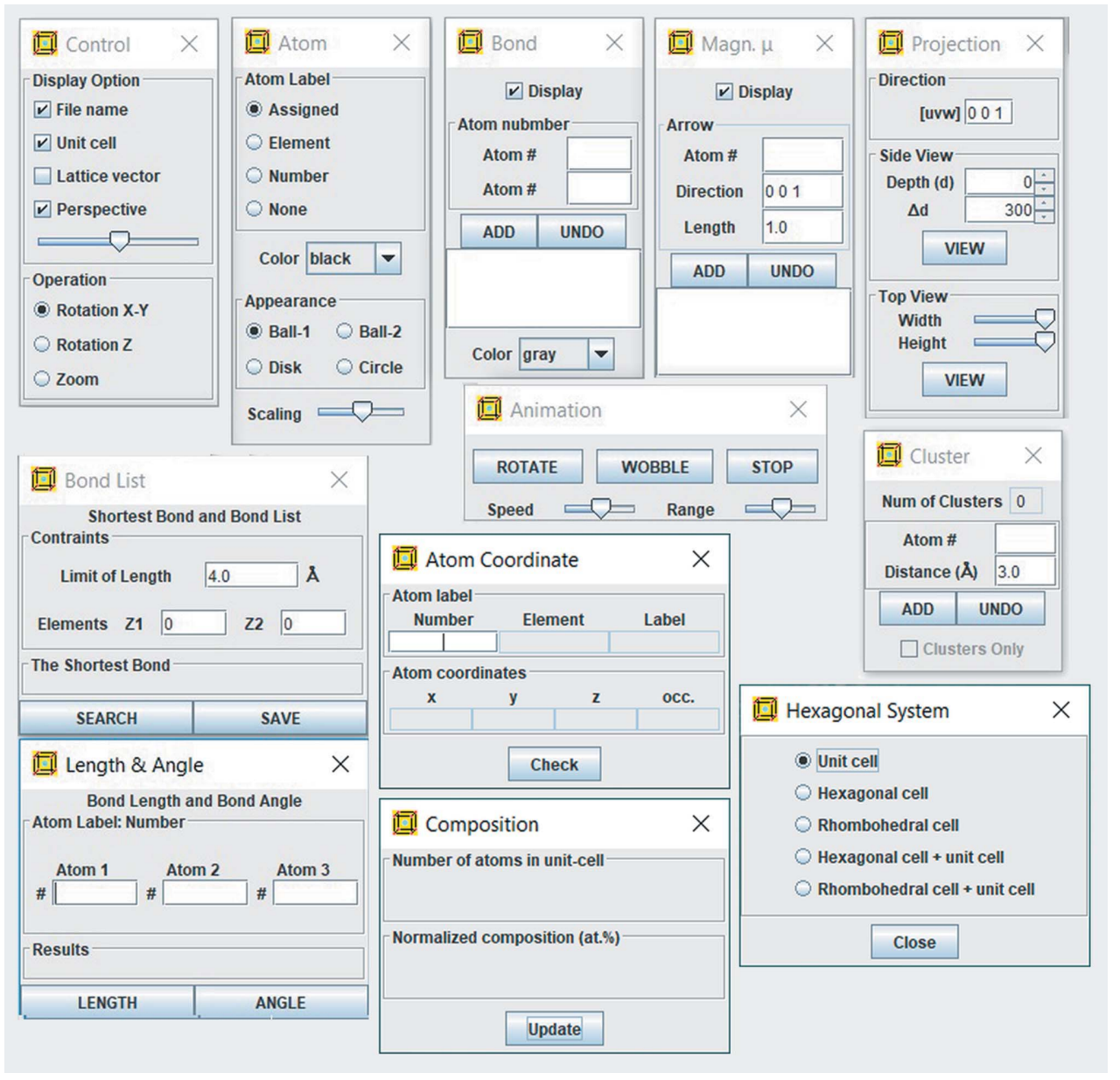

Figure 2

Operational panels of SVAT4 for visualization and analysis. 
structural projection and the polyhedral clusters. Since the input data are the independent atoms in the unit cell, it is practical to save the full list of atoms in the unit cell for further structure modification. Another useful function is the ability to calculate the chemical composition of the crystal structure, which can be used for double-checking of the input data. Hexagonal and rhombohedral structures can be visualized in both conventional and symmetric unit cells. Additional functions are provided for calculation of the bond length/angle for an atom pair/triplet. The structure can be displayed in animated modes of rotation or sway. The crystal structures can be saved as JPEG and TIFF image formats. A unique feature is that the user's customized work on the structure for analysis can be saved to a file, which can be reloaded for a quick display next time or to continue unfinished work.

\subsection{Selected functions}

Details on the functions and usage of SVAT4 are given in the user manual, and selected topics are outlined below:

In $S V A T 4$, the crystal structure may be rotated around the horizontal and vertical axes and the screen normal separately. Thus, the crystal structure may be visualized in an arbitrary orientation by using a mouse (step $1^{\circ}$ ) and by using the arrow keys (step $0.1^{\circ}$ ) or along a specified orientation, i.e. [uvw]. Display of the file name, lattice frame, unit-cell vectors, latticeonly mode, or orthographic or perspective view is optional. A dialog is provided for adjusting the position, font size and color of the file name. Animation can be set up for rotation or sway modes. Crystal structures can be compared in multiple frames of SVAT4 simultaneously.

Atoms can be displayed as circles or discs or two types of solid balls, either translucent or opaque. The radii of atoms are predefined according to the empirically measured and calculated data from Wikipedia (https://en.wikipedia.org/wiki/ Atomic_radius). The radii and colors of atoms can also be revised by users. For example, users may replace atomic radii with ionic radii (https://en.wikipedia.org/wiki/Ionic_radius). Atoms can be tagged with assigned labels or elemental or numeric labels or no labels. The size of the unit cell and overall sizes of all atoms can be adjusted separately. Bonds between atoms or magnetic moments of atoms can be added to the crystal structure. Work on the customized appearance of the crystal structure can be saved and reloaded.

Crystal structures can be analyzed as a projection of the unit cell or a slice of atoms along a selected direction $[u v w]$ and polyhedral atomic clusters, each of them defined by a central atom and a bond distance. Other functions include (i) to calculate the shortest bond and a bond list in a crystal structure; (ii) to retrieve the coordinates of an individual atom; (iii) to calculate the bond distance and angle between two adjacent bonds; and (iv) to retrieve the chemical composition. Users may save the full list of atoms in a unit cell, which can be used for modification of the structure in a new model.

\subsection{Usage}

A template has been designed for the preparation of new structure data with computer assistance, as shown in Fig. 3. In
$S A V T 4$, the standard notations of the 230 space groups in International Tables for Crystallography (Hahn, 2005) are built in, and the Hermann-Mauguin symbols are used. If the crystal structure is described in a non-conventional spacegroup notation, a transformation to the standard space-group notation is required. A function for deciphering CIF format is also available in the template. A collection of crystal structures, taken from De Graef \& McHenry (2012) and from the author's work, was included in the Landyne software suite. The data set includes most of the typical prototypes of crystal phases, and a gallery of the crystal structures is shown online (Li, 2020b). Crystal structures of alloys and intermetallic phases can be retrieved from many resources, e.g. the AFLOW library of crystallographic prototypes (Mehl et al., 2017; Hicks et al., 2019; http://aflowlib.org/CrystalDatabase/), Pearson's Handbook (Villars, 1998) and the International Center for Diffraction Data's Powder Diffraction File (http:// www.icdd.com/).

The use of SVAT4 is mostly straightforward and selfexplanatory. However, some operations may not be so obvious for first-time users. A few examples are listed below. The orientation and zoom operations of the crystal structure can be adjusted with the left mouse button for rotation along the $x-y$ axes, the right mouse button for rotation along the $z$ axis and the mouse wheel for zoom in/out: or using the options for the three operations on the control dialog. The data for a rhombohedral structure can be prepared in two ways: as a hexagonal cell using the $\mathrm{R}$ lattice type in the trigonal system or as a conventional cell using the rhombohedral system. If two

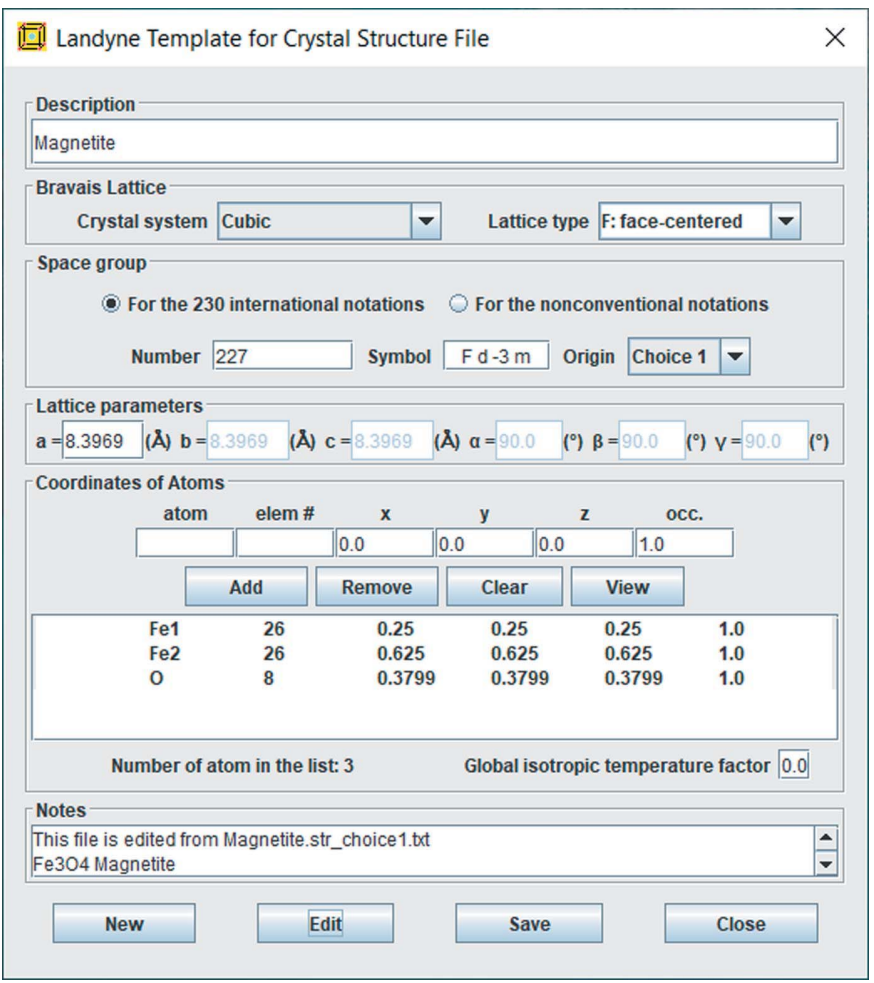

Figure 3

Template for preparation of input data with computer assistance in SAVT4. 
origin choices are available in a space group but the user is uncertain which one should be used, the user may check the chemical composition and bond distance of each structure for verification of the input data. In order to view the structure along a selected direction $[u v w]$, the direction in the 'Layer and Projection' dialog should be selected first, and then the structural view re-selected. When using the arrow keys to adjust the orientation of the crystal structure, make sure that the cursor is over the display panel. In order to display the previous analysis of a structure, the input data of the structure should be loaded first, and then the corresponding work file. (d) a top view of the atom distribution in the flat layer as marked in $(c)$. The polyhedral cluster shows slightly distorted icosahedral coordinates. The flat layer reveals locally fivefold symmetric features.

\subsection{FeSi and CoSi}

FeSi (CoSi) has the B20 prototype structure, one of the fascinating materials in modern condensed-matter physics. The B20 structure has a non-centrosymmetric space group, $198\left(P 2_{1} 3\right)$, which is achiral owing to the neutral $2_{1}$ or $180^{\circ}$

\section{Examples}

This section aims to highlight the use and features of SVAT4 with examples that were chosen from the author's previous research and consulting work.

\subsection{Si and FePt}

Silicon has a diamond cubic crystal structure, $a=0.543 \mathrm{~nm}$ (Ashcroft \& Mermin, 1976). Fig. 4(a) shows the crystal structure of silicon. The nearestneighbor bonds between silicon atoms are shown to emphasize the structural features.

FePt films and FePt-based nanocomposite films containing the highanisotropy tetragonal $\mathrm{L}_{0}$ ordered phase have significant potential for extremely high density perpendicular magnetic recording media (Li et al., 2007). Fig. 4(b) shows the crystal structure of the FePt $\mathrm{L} 1_{0}$ tetragonal phase with the lattice parameters $a=0.385 \mathrm{~nm}$ and $c=$ $0.371 \mathrm{~nm}$; magnetic moments were added on Fe atoms along the [001] zone axis to illustrate the magnetic anisotropy.

\section{2. $\mathrm{Al}_{3} \mathrm{Mn}$}

$\mathrm{Al}_{3} \mathrm{Mn}$ is an orthorhombic intermetallic phase, space group 62 (Pnma), $a=1.487, b=1.247, c=1.256 \mathrm{~nm}$, which is a crystalline approximant of the $\mathrm{Al}-\mathrm{Mn}$ decagonal quasicrystal (DQC). The structural relationship between the $\mathrm{Al}_{3} \mathrm{Mn}$ phase and the $\mathrm{Al}-\mathrm{Mn}$ DQC has been reported in detail on the basis of selected-area electron diffraction (SAED) and high-resolution electron microscopy results (Li, 1995). Fig. 5 shows $(a)$ the crystal structure of $\mathrm{Al}_{3} \mathrm{Mn}$, (b) a polyhedral cluster around an $\mathrm{Mn}$ atom, $(c)$ a side view of the structure with lines marking a flat layer of atoms and
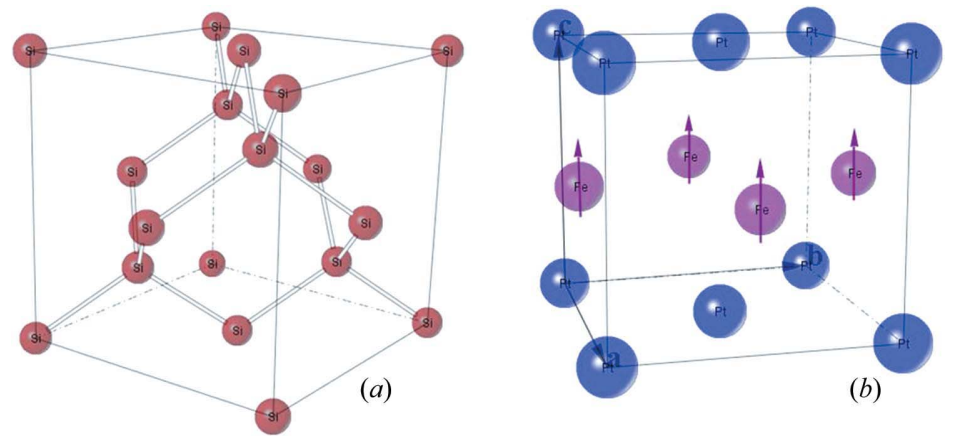

Figure 4

(a) The crystal structure of the silicon phase and (b) the crystal structure of the FePt $\mathrm{L} 1_{0}$ tetragonal phase.

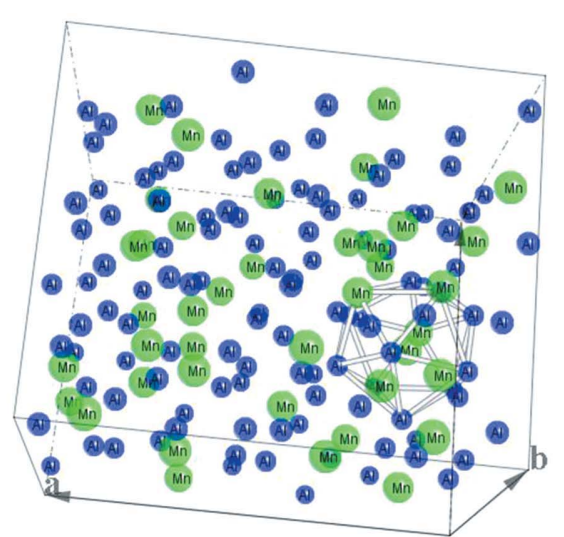

(a)

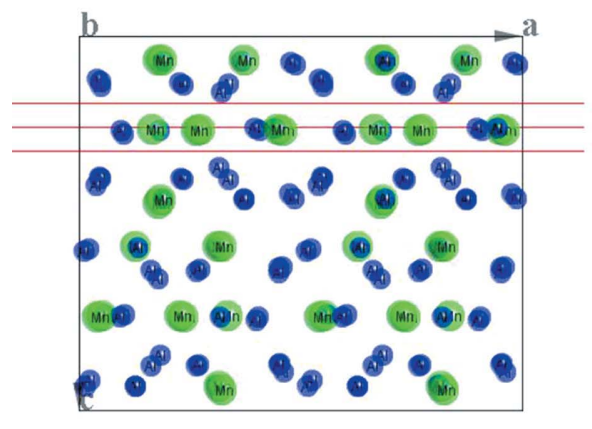

(c)

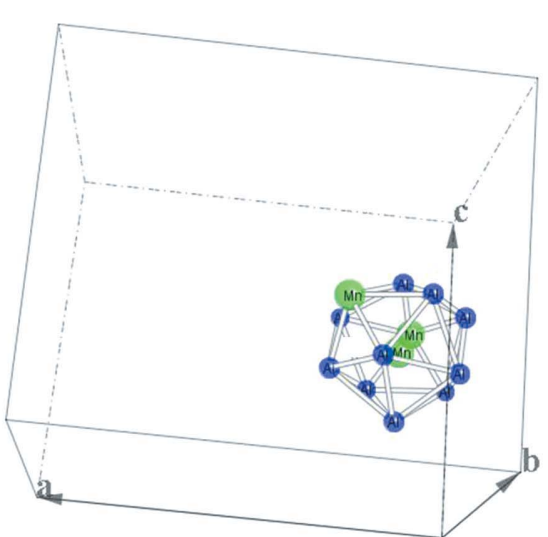

(b)

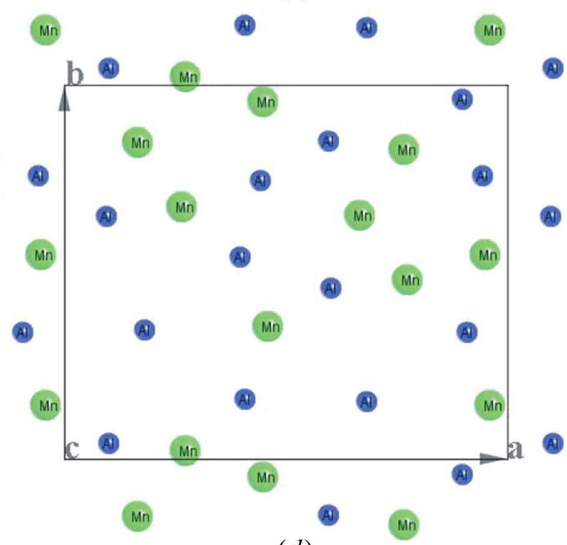

(d)
Figure 5

(a) The crystal structure of $\mathrm{Al}_{3} \mathrm{Mn},(b)$ a polyhedral cluster around an $\mathrm{Mn}$ atom, (c) a side view of the projection with a flat layer of atoms marked and $(d)$ a top view of the atom distribution in the flat layer as marked in $(c)$. 
Table 1

Two sets of the atom coordinate for the FeSi B20 structure.

They are related by the transformation $1 / 4+x, 1 / 4+y, 1 / 4+z$.

\begin{tabular}{llll}
\hline Data set & Fe $x(=y=z)$ & Si $x(=y=z)$ & Reference \\
\hline 1 & 0.38650 & 0.09262 & Wilhelm et al. (2007) \\
2 & 0.13650 & 0.84262 & Wartchow et al. (1997) \\
\hline
\end{tabular}

screw axis. The lattice parameter is $a=0.4489 \mathrm{~nm}$ for FeSi and $a=0.4438 \mathrm{~nm}$ for CoSi. A recent study shows that the chirality in $\mathrm{Co}_{1+x} \mathrm{Si}_{1-x}$ leads to nonzero Dzyaloshinskii-Moriya interactions, which create spin spirals and support skyrmions with broken helicity (Balasubramanian et al., 2020).

Two sets of atom coordinates for the FeSi B20 structure were reported, as shown in Table 1. Fig. 6 shows the crystal structures of FeSi using (a) data set 1 and (c) data set 2. A tetrahedron of three $\mathrm{Si}$ and one $\mathrm{Fe}$ is outlined in Fig. 6(b) to emphasize the structural feature of the B20 structure. The same tetrahedron and three $\mathrm{Fe}$ atoms surrounding the tetrahedron are shown in Fig. $6(d)$, which was constructed using a periodic transformation of the atoms in Fig. 6(c), as indicated by arrows. The atomic coordinates in the two data sets are related by the transformation from $(x, y, z)$ in set 2 to $(1 / 4+x$, $1 / 4+y, 1 / 4+z$ ) in set 1 .

\subsection{As}

Arsenic ( $\alpha$-As) has the A7 prototype structure, space group $166(R \overline{3} \mathrm{~m}), a=0.3760, c=1.0457 \mathrm{~nm}$. The coordinates of the only independent atom are $(0,0,0.2271)$. Other elements, $\mathrm{Sb}$

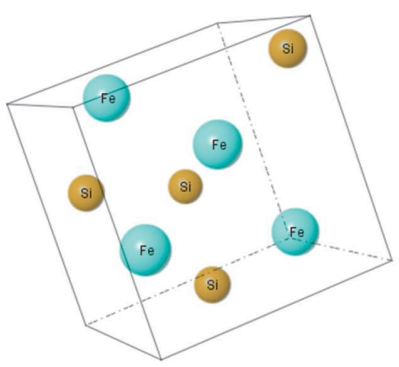

(a)

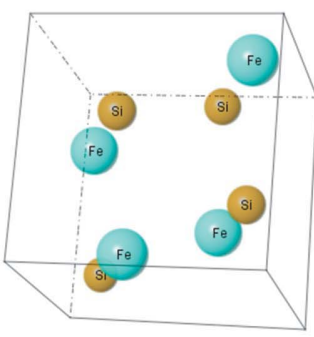

(c)

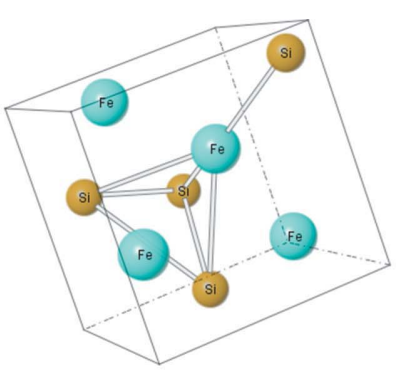

(b)

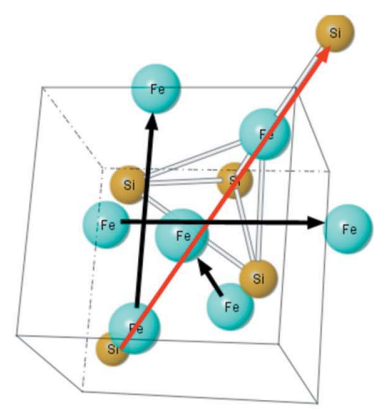

(d)
Figure 6

The crystal structure of the FeSi B20 phase using (a) data set 1 and (c) data set 2 in Table 1. (b) A tetrahedron of three $\mathrm{Si}$ and one Fe is outlined, and $(d)$ the same tetrahedron is shown, with three Fe atoms around the tetrahedron added by using a periodic transformation of the atoms in $(c)$.
Table 2

Two sets of atom coordinate for the $\mathrm{Fe}_{3} \mathrm{O}_{4}$ inverse spinel structure.

Data set 1 corresponds to the second origin choice. Data set 2 corresponds to the first origin choice.

\begin{tabular}{lllll}
\hline Data set & $\mathrm{Fe} 1 x(=y=z)$ & $\mathrm{Fe} 2 x(=y=z)$ & $\mathrm{O} x(=y=z)$ & Reference \\
\hline 1 & $1 / 8$ & $1 / 2$ & 0.2549 & Fleet (1981) \\
2 & $1 / 4$ & $5 / 8$ & 0.3799 & Transformed data \\
\hline
\end{tabular}

and $\mathrm{Bi}$, have the same type of structure. Fig. 7 shows the crystal structures of the $\alpha$-As phase in the $(a)$ conventional cell, (b) symmetric cell and (c) primitive cell. This example shows that SVAT4 provides a variety of ways to display hexagonal and rhombohedral structure. Besides the single-cell mode, as shown in Fig. 7, a combination of two types of cells can also be visualized in SVAT4.

\section{5. $\mathrm{Fe}_{3} \mathrm{O}_{4}$}

The cubic inverse spinel $\mathrm{Fe}_{3} \mathrm{O}_{4}$ is ferromagnetic. The structure has space group $227(F d \overline{3} m), a=0.83941 \mathrm{~nm}$, and two origin choices, the first at $\overline{4} 3 \mathrm{~m}$ and the second at $\overline{3} \mathrm{~m}$. Table 2 lists the structure data of $\mathrm{Fe}_{3} \mathrm{O}_{4}$ in two data sets. They are related by the transformation from $(x, y, z)$ with the second origin choice to $(1 / 8+x, 1 / 8+y, 1 / 8+z)$ with the first origin choice.

Both origin choices can be used to prepare the input data in SVAT4. If the origin choice for the atomic coordinates is wrong, the error can be easily detected by checking the composition. For example, if the atom coordinates in data set 1 are used and the first origin is mistakenly chosen, the composition is $\mathrm{Fe}_{24} \mathrm{O}_{8}$, a wrong composition. By contrast, if the atom coordinates in data set 1 are used and the second origin is correctly chosen, the composition is $\mathrm{Fe}_{24} \mathrm{O}_{32}$, a correct composition. This also works for the combination of data set 2 and the first origin choice.

It is expected that the usability of SVAT4 can be broadened with other components in the Landyne suite. Here is a glimpse of such an extension. Fig. 8 shows $(a)$ a [001] projection of the $\mathrm{Fe}_{3} \mathrm{O}_{4}$ structure by using SVAT4, (b) a SAED pattern

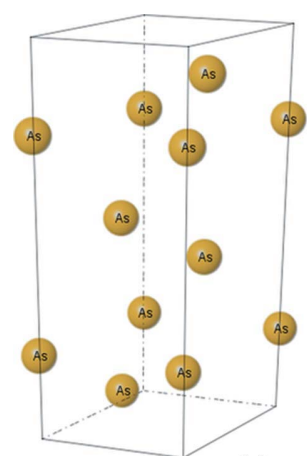

(a)

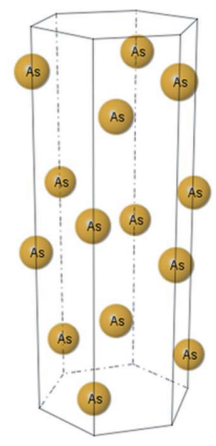

(b)

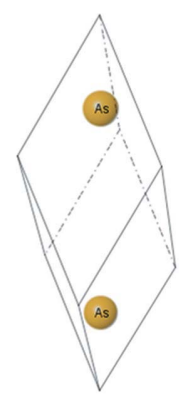

(c)
Figure 7

Crystal structure of the $\alpha$-As A7 phase in the (a) conventional cell, (b) symmetric cell and $(c)$ primitive cell. 


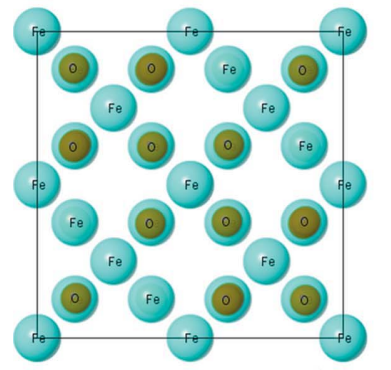

(a)

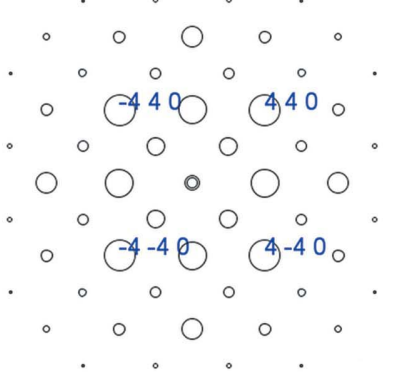

(b)

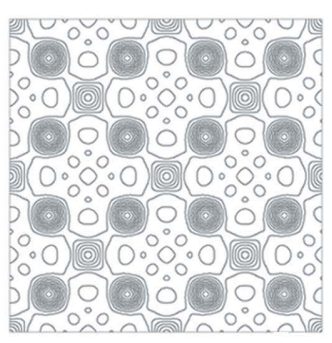

$(c)$

Figure 8

(a) A [001] projection of the $\mathrm{Fe}_{3} \mathrm{O}_{4}$ structure generated from SVAT4, (b) a simulated SAED pattern generated from the SAED4 software, and (c) a projected atom potential map generated from the PAPM software.

simulated using the SAED4 software and $(c)$ a projected atomic potential map obtained using the $P A P M$ software.

\section{Related software}

Data files of crystal structures for SVAT4 are also used in other components in the Landyne suite for simulations and analyses, e.g. SAED4, QSAED3, PCED4, SAKI4 and PAPM. Although the visualization of a crystal structure using $S V A T 4$ is not obligatory before using the data in other components in the Landyne suite, it is recommended to verify the data with $S V A T 4$ first, especially for new input data.

SAED4 is designed for SAED simulation from a single grain in the kinematical and Bloch wave dynamical theories (Li, 2019). Simulations are also possible for composited patterns of twins and multi-phases, and the tool is able to search for the zone axis of an experimental pattern. PCED4 is designed for SAED simulation from polycrystals in the kinematic theory and also in the Blackman theory, a semi-dynamical theory, including peak profiles, textures and mass ratio (Li, 2010). PCED4 provides a straightforward approach for pattern indexing and is very useful for phase identification. SAKI4 is used for simulation and analysis of Kikuchi patterns (Li, 2020a,b). A tool in SAKI4 allows determination of the precise orientation by using an experimental pattern with at least three Kikuchi pairs. $P A P M$ is an extension of $S A E D 4$ and $Q S A E D 3$ for simulation of projected atomic potential (difference) maps $(\mathrm{Li}, 2020 a, b)$. The potential map can be used to correlate to experimental structural high-resolution electron microscopy images and annular bright-field scanning transmission electron microscopy images. The potential difference map can be used to improve the structural model.

\section{Summary}

SVAT4, a component in the Landyne software suite, was developed for visualization and analysis of crystal structures. One unique feature is that the work on structural characterization can be saved and reloaded together with a structure file. The design and functions of SVAT4 have been elaborated, and the usage of SVAT4 was illustrated with selected application examples. Other components in the Landyne software suite can broaden the usability of SVAT4, which is proven to be a useful tool in research and education.

\section{Acknowledgements}

The author would like to thank his colleagues at the Nebraska Center for Materials and Nanoscience, the University of Nebraska-Lincoln, for decades of collaboration, which was the primary motivation for the development of the software. The author would like to thank the reviewers for their suggestions on the improvement of SAVT4.

\section{References}

Ashcroft, N. W. \& Mermin, N. D. (1976). Solid State Physics. New York: Holt, Rinehart, \& Winston.

Balasubramanian, B., Manchanda, P., Pahari, R., Chen, Z., Zhang, W.-Y., Valloppilly, S. R., Li, X.-Z., Sarella, A., Yue, L.-P., Ullah, A., Dev, P., Muller, D. A., Skomski, R., Hadjipanayis, G. C. \& Sellmyer, D. J. (2020). Phys. Rev. Lett. 124, 057201.

De Graef, M. \& McHenry, M. (2012). Structure of Materials: An Introduction to Crystallography, Diffraction and Symmetry, 2nd ed. Cambridge University Press.

Fleet, M. E. (1981). Acta Cryst. B37, 917-920.

Hahn, Th. (2005). International Tables for Crystallography, Vol. A, Space-Group Symmetry. Heidelberg: Springer.

Hicks, D., Mehl, M. J., Gossett, E., Toher, C., Levy, O., Hanson, R. M., Hart, G. \& Curtarolo, S. (2019). Comput. Mater. Sci. 161, S1-S1011. Li, X. Z. (1995). Acta Cryst. B51, 265-270.

Li, X. Z. (2010). Ultramicroscopy, 110, 297-304.

Li, X. Z. (2016). Microsc. Microanal. 22, 564-565.

Li, X. Z. (2019). Microsc. Analysis, May issue, pp. 16-19.

Li, X. Z. (2020a). Software Suite for Electron Diffraction Analysis, https://www.unl.edu/ncmn-enif/xzli/computer-programs.

Li, X. Z. (2020b). Landyne: The Essential Tools for Materials Science Research, http://landyne.scienceontheweb.net.

Li, X. Z., George, T., Jin, L. F., Yan, M. L., Xu, Y. F. \& Sellmyer, D. J. (2007). Microsc. Microanal. 13(S2), 632-633.

Mehl, M. J., Hicks, D., Toher, C., Levy, O., Hanson, R. M., Hart, G. \& Curtarolo, S. (2017). Comput. Mater. Sci. 136, S1-S828.

Villars, P. (1998). Editor. Pearson's Handbook, Crystallographic Data for Intermetallic Phases, Desk Edition. Materials Park: ASM International.

Wartchow, R., Gerighausen, S. \& Binnewies, M. (1997). Z. Kristallogr. New Cryst. Struct. 212, 320.

Wilhelm, H., Schmidt, M., Cardoso-Gil, R., Burkhardt, U., Hanfland, M., Schwarz, U. \& Akselrud, L. (2007). Sci. Technol. Adv. Mater. 8, 416-419. 